\title{
Resinas Ácidas como Catalizadores de la Adición Mucleofílica de Diferentes Alcoholes a Ésteres Grasos Epoxidados
}

* PhD. Profesor - Departamento de Ingeniería Química Universidad de Antioquia - Medellín, Colombia

E-mail: larios@udea.edu.co

** PhD. Profesora Departamento de Ingeniería Química Universidad de Antioquia - Medellín, Colombia

E-mail: gloma@udea.edu.co

$\star \star \star$ Department of Chemical Technology and Heterogeneous Catalysis, RWTH Aachen University, Worringerweg,

Aachen, Germany.

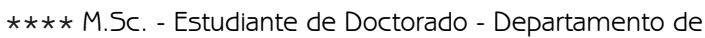
Ingeniería Química - Universidad de Antioquia - Medellín, Colombia

E-mail: ejmms408@udea.edu.co

$\star \star \star \star \star \star$ M.Sc. - Estudiante de Doctorado, Profesor Departamento de Ingeniería Química - Universidad de Antioquia Medellín, Colombia

E-mail: afranco@udea.edu.co

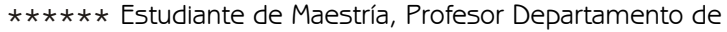
Ingeniería Química - Universidad de Antioquia - Medellín, Colombia

E-mail: erism279@udea.edu.co

Fecha de recepción: Abril 19 de 2004

Fecha de aprobación: Diciembre 17 de 2004

\author{
Luis Alberto Ríos* \\ Gloria Restrepo** \\ Wolfgang F. Hoelderich*** \\ Juan Miguel Marín*** \\ Alexander Franco***** \\ Ricardo Suárez $z^{* * * * *}$
}

\section{RESUMEN}

Se analizó el efecto de varias resinas ácidas como catalizadores para la adición de alcoholes a ésteres grasos epoxidados, usando alcoholes con diferentes configuraciones moleculares $y$ haciendo énfasis en la adición de alcoholes ramificados en las posiciones $\alpha$ y $\beta$. Con respecto a los catalizadores, se determinaron los efectos de la fortaleza ácida y de las restricciones difusionales en la velocidad de reacción y la distribución de productos. Se encontró que la velocidad de reacción se incrementa con la fortaleza ácida pero la selectividad tiene una tendencia contraria cuando se adicionan alcoholes altamente ramificados. La velocidad de apertura del epóxido disminuye al incrementar el número de ramificaciones y el tamaño del alcohol; 
ramificaciones en posición $\alpha$ introducen un mayor impedimento estérico que las ramificaciones en posición $\beta$. Un bajo entrecruzamiento de la resina y/o una alta superficie externa son condiciones necesarias para activar el epóxido cuando se usan resinas basadas en poliestireno como catalizadores. Los productos que se obtuvieron fueron la cetona, proveniente del rearreglo del epóxido, y los trans-ésteres, cuando los tiempos de reacción fueron relativamente largos.

Palabras clave: alcohólisis, ésteres grasos, epóxidos, resinas sulfónicas.

\section{ABSTRACT}

The effects of different alcohol molecular configurations and resin structures were analyzed on the sulfonic acid-resin catalyzed addition of alcohols to epoxidized fatty esters. Emphasis was placed on the addition of $\alpha$-and $\beta$-branched alcohols. Regarding the catalysts, the effect of the acid strength as well as that of the diffusion constraints on the reaction rate and products distributions, were determined. Reaction rate increases with the acid strength but the selectivity shows the opposite trend when highly branched alcohols are added. Epoxide ring-opening rate decreases by increasing the number of branches and size of the alcohol. Branches in $\alpha$ position introduce a higher steric hindrance than branches in $\beta$ position. Low resin crosslinkage and/or high external surface area are necessary conditions to activate the epoxide when polystyrene-based resins are used as catalysts. The obtained byproducts were the ketone, from epoxide rearrangement, and trans-esterified products, when reaction times were relatively long.

Key words: fatty esters, epoxides, alcoholysis, sulfonic resins

\section{INTRODUCCIÓN}

La modificación química de aceites vegetales es una importante ruta para obtener productos industriales usando materias primas renovables [1]. Existe un alto potencial para desarrollar rutas de reacción nuevas que sean eficientes y amigables con el ambiente, conduciendo a nuevos productos, o para encontrar nuevas aplicaciones a productos oleoquímicos existentes. Esta estrategia puede contribuir a disminuir la dependencia de recursos naturales no renovables tales como el petróleo. Una de las funcionalidades que los aceites vegetales ofrecen para modificaciones químicas es el grupo etilénico, el cual puede participar en reacciones tales como acilación, isomerización, dimerización, hidrogenación, hidroxilación, ruptura oxidativa, carboxilación y epoxidación, entre muchas otras $[2,3,4]$. Estas reacciones se caracterizan en general por requerir condiciones severas de reacción y/o reactivos fuertes. La epoxidación merece una atención especial porque posibilita un amplio rango de reacciones que pueden ser realizadas bajo condiciones moderadas de reacción, debido a la alta reactividad del anillo oxirano; por ejemplo, el epóxido puede reaccionar con diferentes nucleófilos para producir mono-alcoholes, dioles, alcoxi-alcoholes, hidroxi-éteres, $M$ hidroxilaminas, mercaptoalcoholes, aminoalcoholes, hidroxinitrilos, etc. [2].

En este trabajo se reportan hallazgos sobre adición de alcoholes al oleato de metilo epoxidado, catalizada por resinas ácidas. Específicamente, se analizó en detalle el efecto de diferentes configuraciones moleculares del alcohol y de resinas con varias estructuras. Aunque la adición de algunos alcoholes primarios lineales a aceites epoxidados ya ha sido reportada previamente $[5,6]$, por ejemplo metanol y butanol, los resultados que aquí se presentan cubren un rango más amplio, y aún no reportado, de alcoholes tales como aquellos ramificados en posiciones $\alpha$ y $\beta$. Las condiciones experimentales y los catalizadores que se usan son también diferentes a los reportados previamente, con lo cual se obtuvieron mejores selectividades hacia los hidroxiéteres bajo condiciones de trabajo más moderadas. 
Los productos de estas reacciones, i.e. hidroxiéteres vecinales, se asemejan a la estructura el aceite de castor, por lo cual podrían encontrar aplicaciones similares, e.g. en lubricantes [7], resinas de poliuretano y de moldeo [8]. La aplicación como lubricante fue la motivación para esta investigación, debido a que los hidroxiéteres tienen la ventaja adicional de que el número de grupos insaturados en el aceite se disminuye y por lo tanto su estabilidad a la oxidación se debería mejorar [9]; además, los grupos funcionales adicionados (hidroxilo y éter) influencian las propiedades físicas de esos lubricantes. Debido a que los alcoholes son nucleófilos muy débiles, su adición a epóxidos demanda la pre-activación del epóxido; por ejemplo, el epóxido puede ser protonado por un ácido Brönsted, en este caso se usaron las resinas ácidas. Subsecuentemente, el alcohol puede atacar al epóxido protonado mediante un mecanismo $S_{N} 2$, tal como se muestra en el esquema 1.

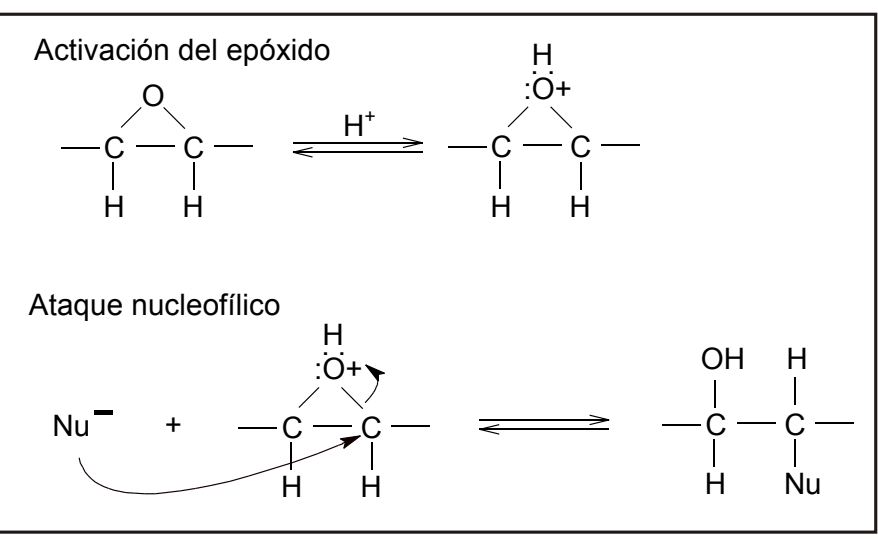

Esquema 1. Ataque nucleofílico sobre un epóxido catalizado por ácidos.

\section{EXPERIMENTAL}

Se usaron cuatro resinas ácidas comerciales como catalizadores: SAC13, Amberlyst 15, Amberlite IR-120 y Dowex50X2; la primera, $5 A C 13$, es un material compuesto hecho de nanopartículas de Mafion atrapadas en una matriz de sílica. Mafion es un copolímero de tetrafluoretileno y perfluor-2-(fluorosulfoniletoxi)propilvinil éter. Las otras resinas son copolímeros sulfonados del estireno y el divinilbenceno (DVB). En todas estas resinas la acidez Brönsted es introducida por grupos de ácido sulfónico $\left(-\mathrm{SO}_{3} \mathrm{H}\right)$ fijados en el esqueleto de la resina.

Las reacciones se efectuaron en reactores de vidrio localizados en un baño de aceite el cual se mantuvo a la temperatura deseada. La agitación se realizó usando cruces magnéticas recubiertas con Teflón. Lo aceites epoxidados se produjeron usando un procedimiento conocido [10] a partir de oleato de metilo obtenido de aceite de colza (57.5 wt.\% metil éster de ácido cis-9octadecenóico, 18.5 wt.\% metiléster de ácido cis,cis-9,12-octadecadienóico, 9.2 wt.\% metiléster de ácido cis,cis,cis-9, 12,15octadecatrienóico, 3.4 wt.\% metiléster de ácido trans-9-octadecenóico, el resto son ésteres saturados) y oleato de metilo enriquecido en el metiléster del ácido oleico obtenido de aceite de girasol (97 wt.\% metil éster de ácido cis-9octadecenóico). Todos los catalizadores fueron desgasificados durante la noche a $120^{\circ} \mathrm{C}$ y bajo alto vacío, y conservados en Argón. El alcohol y el catalizador se mezclaron durante $15 \mathrm{~min}$. a la temperatura deseada para permitir la expansión de la resina, luego se adicionó el epóxido. La cantidad total de acidez Brönsted se determinó intercambiando los catalizadores en una solución acuosa de cloruro de sodio el cual estaba en exceso para asegurar un completo intercambio de $\mathrm{H}^{+}$por $\mathrm{Ma}^{+}$, seguido de una titulación de la fase acuosa con hidróxido de sodio [11].

Las mezclas de reacción se analizaron por cromatografía gaseosa en un equipo Hewlet Packard HP 6890 usando una columna capilar FSSE54 de $60 \mathrm{~m}$. Los productos puros fueron caracterizados por espectrometría de masas (GC Varian 3400 CX, MS Varian Saturn 3 at $70 \mathrm{eV}$ and electron ionization), espectroscopía infrarroja (Nicolet Protégé 460, ventanas de $\mathrm{MaCl}$ ) así como ${ }^{1} \mathrm{H} \quad(300 \mathrm{MHz})$ y ${ }^{13} \mathrm{C}$ MMR (75 $\left.\mathrm{MHz}\right)$ con experimentos dept 90 y 135. 


\section{RESULTADOS}

\section{Efecto de la fortaleza ácida}

SAC13 y Amberlyst 15 fueron seleccionados para evaluar el efecto de la fortaleza ácida en la alcohólisis de los epóxidos. Algunas de las propiedades relevantes de estas resinas, incluyendo la fortaleza ácida, se presentan en la tabla 1. La presencia del altamente electronegativo átomo de Flúor en SAC13 polariza el enlace $\mathrm{O}-\mathrm{H}$ en el grupo sulfonil $y$, por lo tanto, incrementa su fortaleza ácida. Los datos en la tabla 1 muestran que SAC13 es aproximadamente dos veces más fuerte que Amberlyst 15. La figura 1 muestra los resultados de la adición de metanol a oleato de metilo epoxidado, usando SAC13 y Amberlyst 15; el primer catalizador tiene claramente una actividad más alta que el segundo. Cuando la comparación se hace en términos del TOF (turn over frequency), que representa la velocidad de reacción por cada sitio activo $\left(\mathrm{H}^{+}\right)$, entonces la diferencia entre estos catalizadores es aún más grande: a 10 horas de reacción el TOF para SAC13 es $0.97 \mathrm{~min}^{-1}$ mientras que el de Amberlyst 15 es $0.04 \mathrm{~min}^{-1}$. Las energías de activación, calculadas a partir del diagrama tradicional de Arrhenius, son $76.9 \mathrm{KJ} / \mathrm{mol}$ para Amberlyst 15 y $33.9 \mathrm{KJ} / \mathrm{mol}$ para SAC13. Estos valores indican que en verdad los sitios ácidos más fuertes en SAC13 son responsables de su mejor actividad, porque ellos disminuyen la energía de activación a menos de la mitad de la requerida con Amberlyst 15.

Para la adición de alcohol neopentílico, ramificado en posición $\beta$, SAC13 es también más activo que Amberlyst 15, pero en este caso la selectividad hacia el hidroxi-éter es alrededor de un 5\% más alta con el último catalizador, tal como se muestra en la figura 2. Una explicación para este comportamiento puede ser que la acidez más fuerte presente en SAC13 protona muy rápido al
Tabla 1. Características de las resinas ácidas usadas como catalizadores

\begin{tabular}{lcccc}
$\begin{array}{c}\text { Composición } \\
\text { química }\end{array}$ & $\begin{array}{c}\text { Cantidad de } \\
\text { ácido } \\
\text { Brönsted } \\
(\mathrm{meq} / \mathrm{g})\end{array}$ & Fortaleza ácida & $\begin{array}{c}\text { Area } \\
\text { sup. BET } \\
\left(\mathrm{m}^{2} / \mathrm{g}\right)\end{array}$ & $\begin{array}{c}\text { Diámetro } \\
\text { prom. poro } \\
(\AA)\end{array}$ \\
\hline $\begin{array}{c}\text { etrafluoroeteno } \\
\text { perfluoro-2- }\end{array}$ & 0,22 & $\begin{array}{c}\mathrm{Ho} \geq-12\left(96 \% \mathrm{H}_{2} \mathrm{SO}_{4}\right) \\
\Delta \delta=50-51 \mathrm{ppm}^{2}(85 \% \\
\left.\mathrm{H}_{2} \mathrm{SO}_{4}\right)^{*}\end{array}$ & 92 & $10-25$ \\
$\begin{array}{l}\text { uorosulfoni letoxi) } \\
\text { pilvinil éter en silica }\end{array}$ & 4,72 & $\begin{array}{c}\Delta \delta=32,4 \mathrm{ppm}^{*}(45 \% \\
\left.\mathrm{H}_{2} \mathrm{SO}_{4}\right)^{*}\end{array}$ & 51 & $400-800$ \\
\hline $\begin{array}{l}\text { Estireno }+20 \% \\
\text { divinil benceno }\end{array}$ & 4,5 & ibid & $\begin{array}{c}\text { gel } \\
\text { (dilata) }\end{array}$ & No aplica \\
\hline $\begin{array}{l}\text { Estireno }+8 \% \\
\text { divinil benceno }\end{array}$ & 4,3 & ibid & $\begin{array}{c}\text { gel } \\
\text { (dilata) }\end{array}$ & No aplica \\
\hline $\begin{array}{l}\text { Estireno }+2 \% \\
\text { divinil benceno }\end{array}$ & & &
\end{tabular}

${ }^{*} \Delta \delta$ : Diferencia entre los desplazamientos químicos (MMR) de los carbonos $\beta$ y $\alpha$ en la molécula de óxido de mesitilo adsorbida sobre el sitio ácido (14).

epóxido (primera etapa del esquema 1), luego las ramificaciones del alcohol introducen un impedimento estérico que desfavorece el ataque nucleofílico del alcohol (segunda etapa del esquema 1).

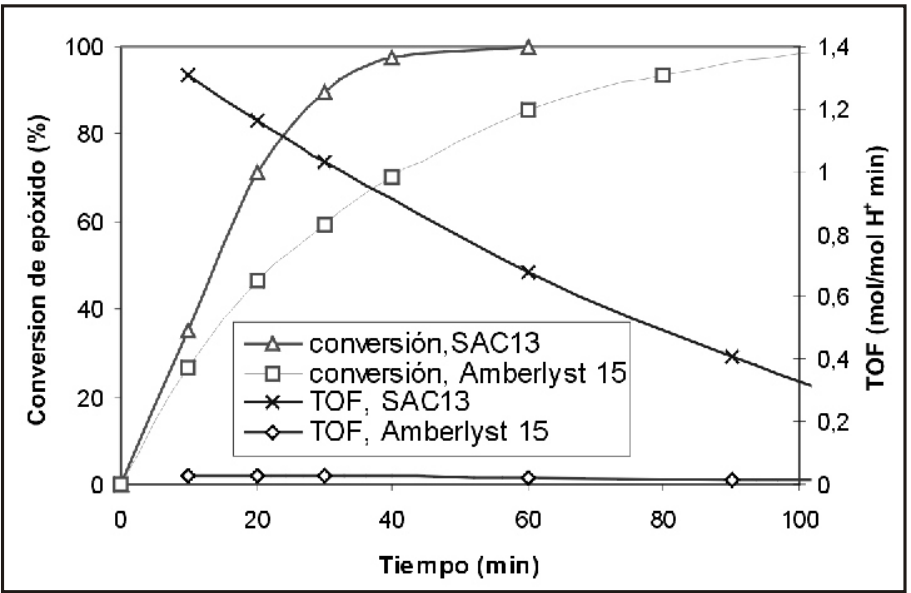

Figura 1. conversión del epóxido y TOF para la adición de metanol a oleato de metilo epoxidado. Alcohol/epóxido $=1$ $\mathrm{g} / \mathrm{g}$, temp. $=60^{\circ} \mathrm{C}$, epóxido/ catalizador $=10$ (masa). Selectividad $>98 \%$ en todos los casos.

En consecuencia, el altamente inestable epóxido protonado se rearregla en una mayor proporción que cuando se adicionan alcoholes pequeños lineales. Más adelante, cuando se presente el efecto intrínseco de la estructura del alcohol, se dará evidencia adicional del impedimento estérico que imponen las ramificaciones. 


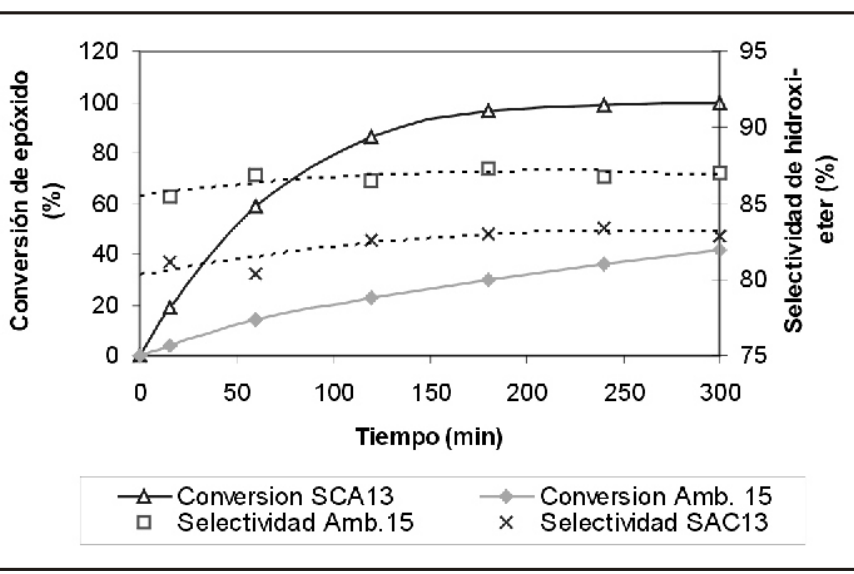

Fig. 2: efecto de la fortaleza ácida en la conversión y distribución de productos para la adición de neopentanol al oleato de metilo epoxidado. Alcohol/epóxido $=10$ (molar), epóxido/resina $=10$ (masa), temp. $=60^{\circ} \mathrm{C}$

\section{Efecto del entrecruzamiento de la resina:}

Las resinas ácidas basadas en poliestireno se producen comercialmente con diferentes contenidos de divinilbeceno (DVB). Al cambiar la cantidad de DVB se cambia el grado de entrecruzamiento, de la resina y por lo tanto las propiedades de dilatación son ajustadas; un bajo contenido de DVB produce un menor entrecruzamiento el cual conduce a una alta dilatación de la resina en un medio polar. Para determinar el efecto del grado de entrecruzamiento de la resina, además de Amberlyst 15 se estudiaron otras dos resinas basadas en ácido sulfónico-poliestireno: Amberlite IR-120 y Dowex50X2; las propiedades de estas resinas se incluyen en la tabla 1.

Se seleccionó la adición de isobutanol al oleato de metilo epoxidado para estudiar el efecto del entrecruzamiento de la resina; los resultados se presentan en la figura 3. Para efectos de comparación se incluyen también los resultados con SAC13.

El isobutanol fue escogido porque la presencia de una ramificación en la posición suministra valiosa información sobre la adición de alcoholes con estructura similar, lo cual fue una de las principales motivaciones para este trabajo. Hay dos aspectos interesantes para discutir: (a) con las resinas microreticulares, i.e. aquellas en la forma de gel, al incrementar el entrecruzamiento se reduce dramáticamente la actividad y (b) la resina macroreticular es bastante activa a pesar de tener un entrecruzamiento mayor que la resina microreticular más entrecruzada. Estos dos aspectos se explican en términos de la accesibilidad del sitio activo; un incremento en el contenido de divinilbenceno disminuye la dilatación de la resina y por lo tanto los sitios ácidos son menos accesibles para el voluminoso substrato (oleato de metilo epoxidado), el cual tiene un tamaño molecular de aproximadamente $10 \AA$. La resina macroreticular (Amberlyst 15) es activa gracias a su relativamente grande superficie externa (ver tabla 1), los protones localizados sobre la superficie externa parecen ser aquellos catalizando la alcohólisis ya que los interiores son inaccesibles debido al alto entrecruzamiento.

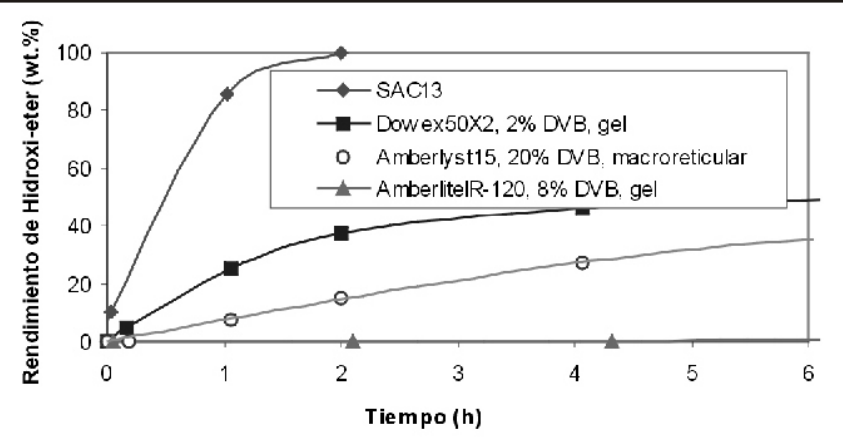

Fig. 3: influencia del entrecruzamiento de la resina y de su estructura en la adición de isobutanol a oleto de metilo epoxidado. Alcohol/epóxido = 10 (molar), epóxido/resina $=10$ (masa), temp. $=40^{\circ} \mathrm{C}$. Selectividad $>98 \%$ en todos los casos.

SAC 13 es muy activa por las razones ya descritas, pero su alto valor comercial, junto con el hecho de que algunas resinas más baratas basadas en poliestireno muestran buena actividad, conduce a escoger esta última para los estudios siguientes y para la producción a mayor escala. Además, cuando se estudiaron alcoholes altamente ramificados, como el neopentanol, la altamente acídica SAC13 condujo a menores 
selectividades en comparación con las resinas de poliestireno. Entre estas resinas de poliestireno, se seleccionó Amberlyst 15 porque además de su relativo buen desempeño catalítico, tiene una estabilidad mecánica superior y un tamaño de partícula más grande que facilita su recuperación.

\section{Sistema de reacción y adición de diferentes alcoholes:}

Uno de los principales objetivos fue optimizar la producción de hidroxi-éteres formados a través de la adición de alcoholes a aceites epoxidados. Para hacer esto, se evaluaron varios parámetros de reacción: solvente, temperatura, tiempo y las relaciones alcohol/epóxido, epóxido/catalizador. Mo es la intención de este documento presentar en detalle los resultados de dicha evaluación, que son muy importantes para la producción en gran escala. Como un ejemplo que ayudará a visualizar la distribución de productos, en la figura 4 se presenta el efecto de la temperatura en la adición de neopentanol.

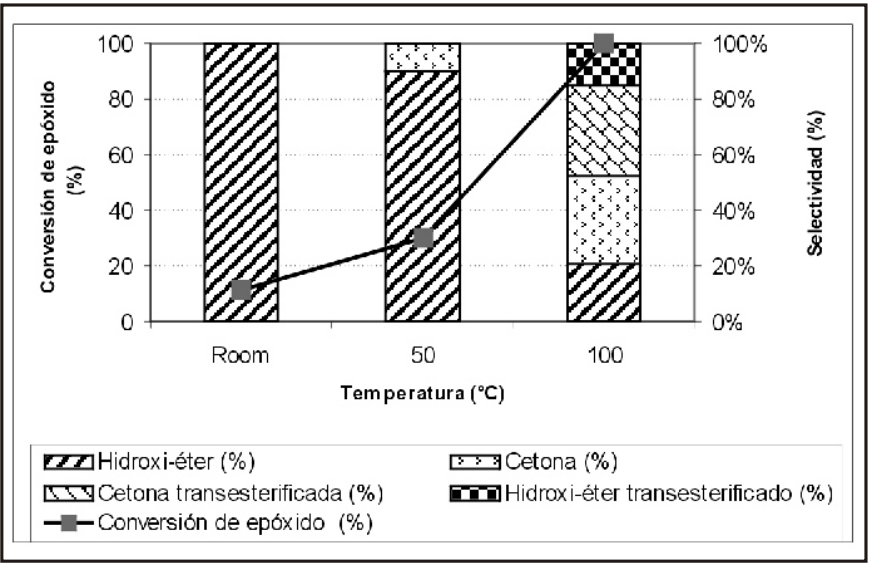

Fig. 4: efecto de la temperatura en la conversión y distribución de productos para la adición de neopentanol a oleato de metilo epoxidado. Alcohol/epóxido $=20$ (molar), epóxido/Amberlyst $15=10$ (masa), 13.2 wt \% tolueno, tiempo $=17 \mathrm{~h}$.

Los resultados de esta figura, junto con otros obtenidos en la adición de diferentes alcoholes, permitieron determinar la ruta de reacción descrita en el esquema 2. A partir del epóxido y el alcohol la reacción se lleva a cabo mediante dos rutas competitivas: rompimiento del anillo epóxico por el alcohol conduciendo a dos regioisómeros y rearreglo del epóxido para producir la cetona, produciendo también dos regioisómeros. Una vez estos productos se han formado, reacciones consecutivas de transesterificación pueden ocurrir, especialmente cuando el tiempo de reacción es lo suficientemente grande. La formación de las cetonas fue un nuevo e interesante hallazgo, el cual hasta donde se ha podido averiguar no ha sido reportado que ocurre con aceites vegetales epoxidados. Ajustando las condiciones de reacción fue posible dirigir la reacción hacia cada uno de los posibles productos, los cuales después de su aislamiento fueron identificados y caracterizados por ${ }^{1} \mathrm{H}$ y ${ }^{13} \mathrm{C}$ MMR así como por las técnicas FT-IRyMS.

La optimización de las condiciones de reacción para maximizar los rendimientos de los hidroxiéteres condujo a los resultados que se presentan en la figura 5, para la adición de varios alcoholes lineales y ramificados en posiciones $\alpha$ y $\beta$. Los alcoholes lineales pueden ser adicionados con muy buenas selectividades hacia los hidroxiéteres bajo condiciones de reacción muy moderadas, pero la introducción de ramificaciones en posiciones $\alpha$ y $\beta$ conduce a una disminución en la selectividad y a condiciones de reacción más demandantes, e.g. se requieren relaciones alcohol/epóxido más altas y tiempos de reacción mayores. El principal producto que se obtuvo en estos experimentos fue la cetona.

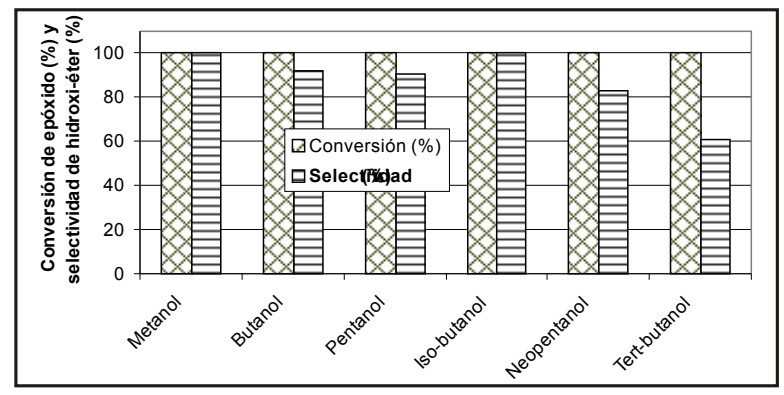

Fig. 5: mejores resultados para la adición de alcoholes a oleato de metilo epoxidado. Metanol, butanol, pentanol, isobutanol/epóxido = 10 (molar). Meopentanol, tert-butanol/ epóxido $=15$ (molar). Epóxide/Amberlyst $15=10$ (masa). Temp. $=60^{\circ} \mathrm{C}$. Tiempo: $1 \mathrm{~h}$ (metanol), $24 \mathrm{~h}$ (butanol y pentanol), $37 \mathrm{~h}$ (isobutanol), $52 \mathrm{~h}$ (neopentanol) y $48 \mathrm{~h}$ (tert-butanol). 


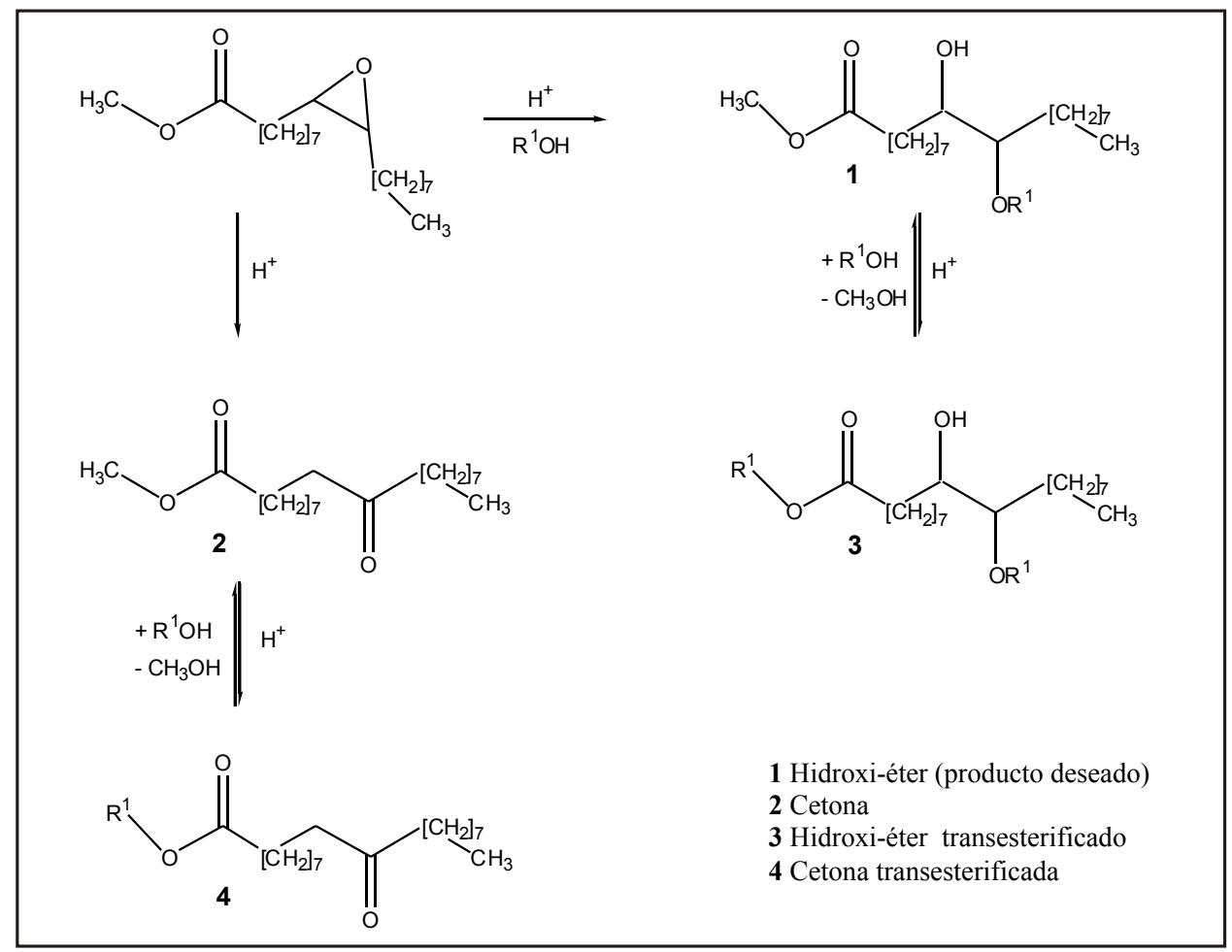

Esquema 2: sistema de reacción para la reacción de alcoholes con oleato de metilo epoxidado

Efecto intrínseco de la estructura del alcohol:

Con el fin de comparar verdaderamente el efecto de la estructura del alcohol en la velocidad de reacción y en la selectividad, las reacciones tienen que ser llevadas a cabo bajo las mismas condiciones de reacción, por lo tanto, se tuvo que realizar una serie de experimentos para determinar las mejores condiciones de reacción para realizar la reacción más difícil. La reacción más difícil fue la adición de alcohol neopentílico, debido al impedimento estérico impuesto por sus ramificaciones y al hecho de que el punto de fusión de este alcohol es relativamente alto $\left(52^{\circ} \mathrm{C}\right)$. El neopentanol es sólido a la temperatura requerida para evitar el rearreglo del epóxido, por lo tanto fue necesaria la adición de un solvente.

Otros dos alcoholes que tienen la estructura neopentílica fueron incluidos en esta comparación, debido a que uno de los principales objetivos del proyecto en el que se enmarca este trabajo es la adición de este tipo de alcoholes. Es así como 3-benciloxi-2,2-dimetilpropanol y 3-(2'- etil)-butiloxi-2,2-dimetilpropanol fueron sintetizados con rendimientos del $81 \%$ and $13 \%$ (basados en el dioxano) respectivamente, siguiendo el procedimiento descrito en una patente [12]. Los reactivos para estas reacciones fueron los dioxanos producidos por la reacción de un aldehído con neopentilglicol [13]. Los productos finales, i.e. los neoalcoholes, fueron purificados mediante destilación a alto vacío.

La figura 6 muestra los resultados de la adición de diferentes alcoholes al oleato de metilo epoxidado bajo condiciones de reacción en las que se evita la formación de cetonas. De esta figura es evidente el papel que juega la estructura del alcohol en la reacción; las velocidades de reacción, según el tipo de alcohol, están en el orden:

Alcohol lineal >alcohol ramificado en $\beta>$ alcohol ramificado en $\alpha>$ alcohol ramificado en $\beta$ con sustituyentes voluminosos. 


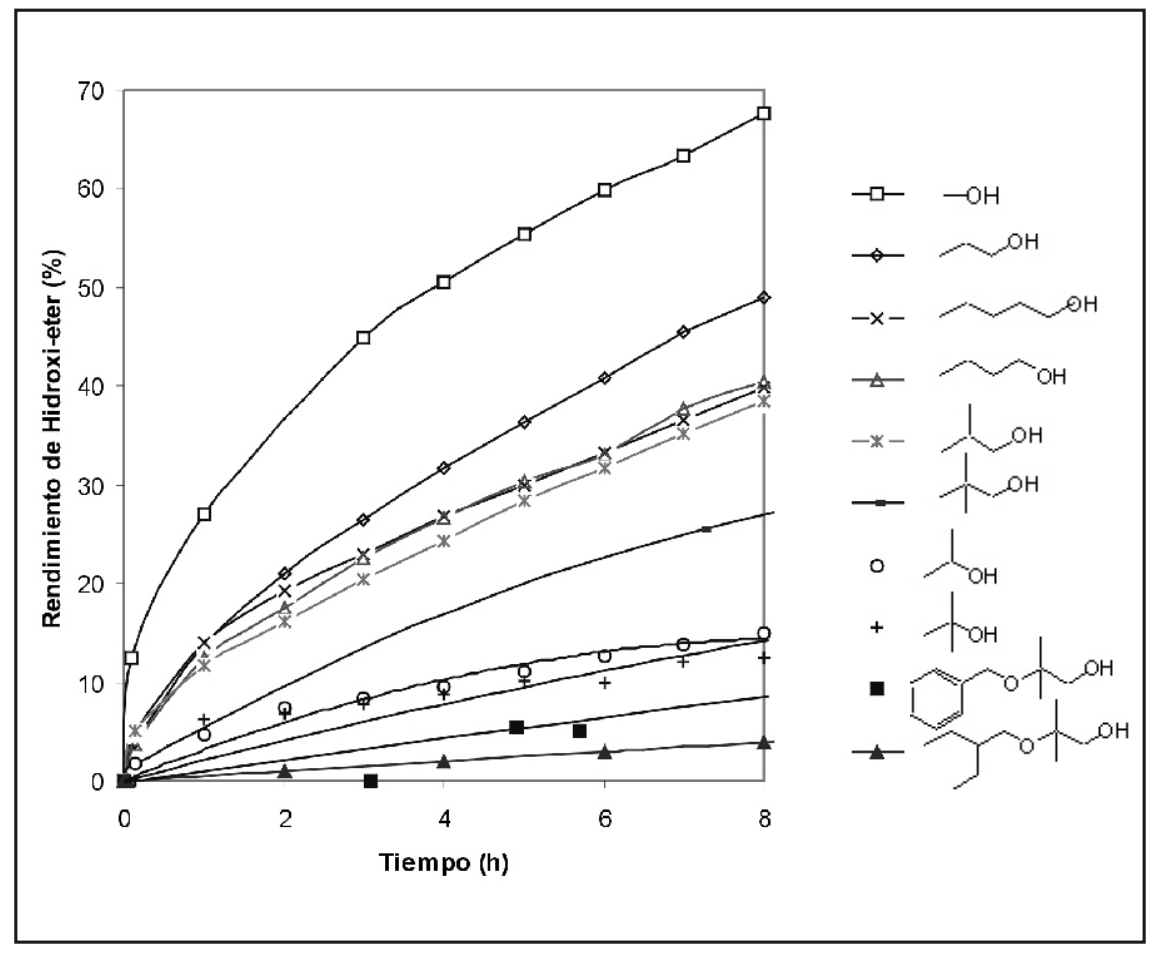

Fig. 6: efecto de la estructura del alcohol en la velocidad de alcohólisis. Alcohol/epóxido = 5 (molar), epóxido/Amberlyst $15=2.5$ (masa), tolueno/epóxido $=2$ (masa), temp. $=$ $24^{\circ} \mathrm{C}$. Selectividad $>98 \%$ en todos los casos.

Dentro de cada una de las familias anteriores la velocidad de reacción disminuye con el número de ramificaciones y con su tamaño. Se puede notar que en los dos éter-alcoholes el oxígeno del éter contribuye a la baja reactividad con su efecto inductivo (atracción de electrones). Para revisar que la jerarquía de actividad no es una consecuencia de algunas estricciones impuestas por la resina utilizada (Amberlyst 15, 20\% DVB), también se realizaron experimentos usando una resina menos entrecruzada (Dowex50X2, 2\% DVB); con esta resina se conservó el orden de actividad $y$, como era de esperarse, las velocidades de reacción fueron mayores. Aunque Amberlyst 15 y Dowex50x2 tienen un número similar de sitios $\mathrm{H}^{+}$, solamente aquellos localizados en la superficie externa de Amberlyst 15 están actuando, los interiores no son accesibles debido al alto entrecruzamiento de ésta, esto explica la menor actividad observada con Amberlyst 15.

\section{CONCLUSIONES}

Las resinas ácidas son catalizadores heterogéneos adecuados para la adición de alcoholes a ésteres grasos epoxidados, siempre y cuando los sitios ácidos sean accesibles para activar al epóxido. La velocidad de reacción se incrementa con la fortaleza ácida pero esta a su vez conduce a una menor selectividad cuando se utilizan alcoholes altamente ramificados. Resinas basadas en poliestireno que tienen un contenido de DVB mayor del $8 \%$ son demasiado entrecruzadas para permitir el contacto entre el epóxido y los sitios ácidos interiores, por lo tanto, para observar actividad deben estar presentes suficientes sitios ácidos en la superficie externa, tal como en el caso de Amberlyst 15 . El principal subproducto obtenido fue la cetona formada como dos regioisómeros mediante la reacción competitiva de rearreglo del epóxido. Cuando los tiempos de reacción son relativamente grandes también se obtienen los productos transesterificados. La velocidad de apertura del anillo oxirano disminuye al incrementar el número de ramificaciones y el tamaño del alcohol; ramificaciones en posición $\alpha$ introducen un mayor impedimento estérico que ramificaciones en posición $\beta$.

\section{AGRADECIMIENTOS}

Los autores agradecen a la Fundación Alemana para la Ciencia (Deutsche Forschungsgemeinschaft, proyecto SFB 442) y a la Universidad de Antioquia por el soporte financiero e infraestructura para desarrollar este trabajo. 


\section{REFERENCIAS}

[1] K. Hill, J. Oleo. Sci. 5 (2001) 433.

[2] H. Baumann, M. Bühler, H. Fochem, F., Hirsinger, H. Zoebelein, J. Falbe, Angew.

Chem. 100 (1988) 41.

[3] U. Biermann, W. Friedt, S. Lang, W. Lühs, G. Machmüller, J. Metzger, O. Rüsch, M. Klaas, H.J. Schäfer, M.P. Schneider, Angew. Chem. 112 (2000) 2292.

[4] W.F. Hölderich, U. Keller, J. Fischer, W. Dott, H. Murrenhoff, o+p Ölhydraulik und Pneumatik, 44 (2000) 240.

[5] B. Dahlke, 5. Hellbardt, M. Paetow, W.H. Zech, J. Am. Oil Chem. Soc.72 (1995) 349.

[6] A. Corma, F. Martínez, P. Ciudad, Patent W09856780A1: Process and catalyst for the production of epoxides and hydroxylated derivatives of acids and esters of fatty acids. (1998).

[7] S. Asadauskas, J.M. Perez, J.L. Duda, J. Soc. Tribo. and Lub. Eng. 12 (1997) 35.

[8] B. Gruber, R. Höfer, H. Kluth, A. Meffert, Fat Sci. Technol. 89 (1987) 147.

[9] E.M. Frankel, Prog. Lipid Res. 19 (1980) 1.

[10] J.G. Wallace, in Kirk Othmer "Encyclopedia of Chemical Technology", $3^{\text {rd }}$ ed., vol. 9, John Wiley \& Sons, Mew York, 1978, p.263.

[11] K. Tanabe, in "Solid acids and bases", Academic Press, Kodansha, 1970, p.23.

[12] W. Otte, M. zur Hausen, patent DE 3328561 A1, (1985).

[13] F.A.J. Meskens, Synthesis, (1981) 501.

[14] D. Farcasiu, A. Ghenciu, G. Marino, K.D. Rose, J. Am. Chem. Soc. 119 (1997) 11826. 